\title{
The Cost-Effectiveness of Thrombolysis Administered by Paramedics
}

\author{
Paul A Scuffham * \\ Vivienne Tippett **
}

\section{Affiliations:}

* School of Medicine, Griffith University

${ }^{* *}$ Australian Centre for Prehospital Research, Queensland Ambulance Service

\section{Address for correspondence:}

Paul A Scuffham (PhD)

Professor of Health Economics

School of Medicine

Logan Campus L03 2.43

Griffith University

Meadowbrook 4131

Queensland, Australia

Ph: $+61(0) 733821367$

Fax: +61 (0)733821338

Email: p.scuffham@griffith.edu.au

Date: $\quad$ May 2008

Words in main body of text: 5411

Manuscript type: Full-length original article

This study was presented at the Australian College of Ambulance Professionals conference in Gold Coast, Australia, 26-29 September 2007. 


\section{ABSTRACT}

\section{Objective}

The objective of this study is to estimate the expected health outcomes, costs, cost-effectiveness of changing from Current Practice where thrombolytic therapy is given in hospital to Paramedic Practice where thrombolytic therapy is administered by appropriately trained paramedics (pre-hospital).

\section{Methods}

A decision-analysis microsimulation model was constructed with a 30-day component and a long-term health state transition component. A brief review of the literature was undertaken to obtain data on time to needle to populate the model. The primary health outcome was quality-adjusted life years (QALYs); secondary outcomes included cardiac events, procedures, and survival. Costs to the Australian healthcare system for the rest of life were taken as the analytical perspective.

\section{Results}

On average, STEMI patients gain 0.13 QALYs at an additional life-time cost of $\$ 343$. The incremental cost-effectiveness ratios were $\$ 3428$ per life-year gained and $\$ 2601$ per QALY gained. These estimates were robust to changes in a range of assumptions and parameter values. The most important factor was the time-to-needle the greater the difference between Current Practice times and Paramedic Practice times, the greater the health benefits and lower the cost per QALY (and life-year) gained. A key factor in the model was the substantially lower incidence of heart failure from earlier time-to-needle. Importantly, there was little change in the cost per QALY gained for a 
wide range of ages; thus, there is no argument to limit thrombolysis by paramedics to above or below an age threshold.

\section{Conclusions}

Paramedics administering thrombolysis can avert some STEMI deaths and the pre-hospital administration of thrombolysis is good value for money.

Key words: STEMI, microsimulation, cardiac death, time to needle, pre-hospital care

Short title: Cost-effectiveness of paramedic thrombolysis 


\section{INTRODUCTION}

Thrombolytic therapy is one of the most effective treatments ever developed for a condition that kills more people globally than any other. Following the development of $1^{\text {st }}$-generation thrombolytic therapy (i.e. streptokinase) and $2^{\text {nd }}$-generation thrombolytic therapy (i.e. front-loaded (accelerated) alteplase), the $3^{\text {rd }}$-generation of thrombolytic agents have advanced to recombinant tissue plasminogen activators (t-PA) (reteplase and tenecteplase). These fibrin-specific agents are indicated up to 12 hours after onset of symptoms, whilst earlier generation drugs (alteplase) provided benefit in a shorter six hour window following onset. The newest agents are administered as a single IV bolus injection. The ASSENT-2 ${ }^{1}$ trial showed tenecteplase has survival gains identical to alteplase and a small, but significantly lower rate of bleeding and heart failure compared to alteplase, and in non-inferiority trials, reteplase has been shown to be at least as effective as streptokinase ${ }^{2}$. Meta-analyses have shown no significant difference between thrombolytics in 30-35 day mortality ${ }^{3}$; however, that the risk of a major bleeds and anaphylaxis is higher with streptokinase compared with alteplase, reteplase and tenecteplase, whereas the risk of intracranial haemorrhage and stroke is greater for the newer drugs compared with streptokinase ${ }^{4}$. This risk is stroke was not statistically different between alteplase, reteplase and tenecteplase ${ }^{4}$. Currently, the fibrin-specific agents (tenecteplase and reteplase) are considered to be the thrombolytics of choice in Australia and Scotland ${ }^{5}$. However, these thrombolytics are relatively expensive at approximately $\mathrm{A} \$ 1,300$ per dose $(\approx \cup S \$ 1,000)$. Therefore, timely use of these thrombolytics is an important factor to maximise the benefit.

It is well established that time-to-needle (TTN) is an important factor in the management of acute myocardial infarction (AMI) patients to enhance the chance of 
survival. Clinical trials have shown substantial short-term and long-term survival gains resulting from earlier administration of thrombolytic therapy. For example, the 2-year survival rates for those treated within 70 minutes of symptom onset was $98 \%$ compared with $88 \%$ for those who received treatment after 70 minutes $^{7}$; a pooled analysis showed a $17 \%$ reduction in mortality from administering thrombolysis pre-hospital (time-toneedle: 104 minutes vs 162 minutes in hospital) ${ }^{8}$; and a further study showed delaying thrombolysis by 60 minutes increases the 5 -year mortality hazard ratio by $20 \%{ }^{9}$.

The UK's National Service Framework for Coronary Heart Disease states that "thrombolysis should be given within 60 minutes of calling for professional help" ${ }^{10}$. The Australian Guidelines for managing acute coronary syndromes recommend thrombolysis is the preferred treatment within one hour of symptom onset; percutaneous coronary intervention $(\mathrm{PCl})$ is preferred between 1-3 hours but if thrombolysis can be given onehour earlier or if transport to hospital is delayed by more than 30-minutes then thrombolysis is preferred; and $\mathrm{PCl}$ is the recommended intervention if three hours or more have elapsed from symptom onset ${ }^{5}$.

In Queensland, thrombolytic therapy is primarily administered in emergency departments. However, the time from symptom onset to calling for assistance is, on average, 43 minutes, and from calling for professional help to receiving thrombolytic therapy an additional 83 minutes (i.e. 126 minutes in total) ${ }^{11}$. This time includes the time for paramedics to travel to the patient, assessment of signs and symptoms (including a 12-lead ECG), preliminary diagnosis of ST-elevated myocardial infarction (STEMI), transport the patient to the emergency department, hand-over, duty clinician treatment decision and finally the instigation of thrombolytic therapy. (A STEMI indicates an occlusive thrombosis in an epicardial coronary artery). This TTN could be reduced 
substantially (by approximately 52 minutes) by allowing paramedics to administer thrombolytic therapy after making a preliminary diagnosis and before transportation to the emergency department. Thus, the TTN administered by paramedics would be less than 30 minutes from calling for assistance, and in most cases thrombolysis could be given at least one hour before $\mathrm{PCl}$ could be undertaken ${ }^{5}$. This reduced TTN might reduce deaths, length of stay in hospital, reduce the severity of consequences of AMI (e.g. moderate to severe heart failure classified as NYHA II $-\mathrm{IV}^{12}$ ) and reduce the need for associated procedures (such as coronary artery bypass grafts). Therefore, reducing the time-to-needle will result in better health outcomes to patients and might provide cost-savings to the health system.

The objective of this study is to estimate the expected health outcomes, costs, and cost-effectiveness of changing from Current Practice where thrombolytic therapy is given in hospital to a practice where thrombolytic therapy is administered by paramedics (pre-hospital). We rely on data from large thrombolytic trials ${ }^{13-19}$ and meta-analyses of the efficacy and outcomes of thrombolytics ${ }^{32021}$ and clinical trials that have specifically examined the effect of time-to-needle and/or prehospital thrombolysis ${ }^{8921-25}$. In addition, tenecteplase is selected as the thrombolytic for all comparisons as use of this thrombolytic has recently been implemented by paramedics in Queensland.

\section{METHODS}

A cost-utility analysis was undertaken in a decision analysis framework. A microsimulation model with health-state transition processes was developed to estimate the cost-effectiveness of thrombolysis administered by paramedics (Paramedic Practice) compared with thrombolysis given in emergency departments of public hospitals (i.e. 
Current Practice) for patients with STEMI. The primary health outcome used in this analysis is the quality-adjusted life year (QALY) to combine various health outcomes, events and survival into an index. Secondary outcomes include life-years (survival unadjusted for quality of life) and numbers of events (e.g. coronary interventions, strokes, cases of heart failure and deaths). This study focuses on the direct healthcare costs incurred by the Government for STEMI. Although costs to the patient and/or health insurer may be not insignificant, they are excluded from this analysis as the majority of costs for acute care are borne by the public sector.

\section{The Model}

The model builds on earlier work by Kellet ${ }^{26}{ }^{27}$ constructed using TreeAge Pro ${ }^{28}$. There are two components to the model - the consequences at 30-days (acute phase) and the longer-term consequences. The model considers only those patients who have the confirmed diagnosis of STEMI.

The clinical pathways in the acute phase of the model were developed to include all feasible events. There are five primary events that may occur within the acute phase (MI death, anaphylaxis, haemorrhage, stroke, heart failure) giving a core of 31 possible pathways through the acute phase depending on which events occur. In addition to these core events, additional events and interventions may occur depending on the preceding events. For example, the patient could die from the MI. If they survive the MI, they could experience an anaphylactic reaction to the thrombolytic treatment. Next, they could experience a major haemorrhage. If they survive the haemorrhage they could suffer a stroke and may or may not die. If they survive a stroke they could experience heart failure. Finally, those who have not experienced a stroke or heart failure could 
require early (within 30 days of the STEMI) surgical revascularisation either by $\mathrm{PCI}$ or CABG. To simplify the pathways we assumed that no elective revascularisation procedures were performed for stroke or heart failure patients.

If the patient survives all the possible hazards during the acute phase, they enter the longer-term component which is modelled as a health state transition process ${ }^{29}$. There are five core health states in this component of the model: dead, alive in good health post AMI, alive with a disabling stroke, alive with $\mathrm{CHF}$, and alive with both a disabling stroke and CHF. Included in this component are subsequent surgical revascularisation procedures $(\mathrm{PCl}, \mathrm{CABG})$ and resynchronisation therapies (pacemakers and implantable cardioverter defibrillators) for those with heart failure. To simplify the model, it was assumed that no further revascularisation procedures are undertaken following a CABG; it was also assumed that implantation of resynchronisation devices (pacemaker or ICD) can be undertaken once only in those with heart failure. Figure 1 presents a simplified depiction of the health state transition process.

FIGURE 1 GOES ABOUT HERE

\section{Intervention and Effectiveness Data}

Time to thrombolysis

The Queensland Ambulance Service (QAS), using data on 28,298 chest pain calls in 2003, report the mean times for various events in the pathway from symptom onset to receiving thrombolysis using an intent-to-treat design (Table 1$)^{11}$. These data 
were obtained from both urban and rural settings across the state. Thrombolytics could be administered by paramedics during the "on-scene" time before transportation to hospital, reducing the TTN from 126 minutes to 74 minutes.

\section{TABLE 1 GOES ABOUT HERE}

\section{Effectiveness of thrombolysis}

Data on the effectiveness of tenecteplase were drawn from a recent health technology assessment ${ }^{4}$. However, preliminary analysis revealed that the probability of death from AMI, the differential timing of administering thrombolytics, and the probability of heart failure (HF) were key factors affecting the ICER. Therefore, a brief review of the literature was undertaken by searching Medline and bibliographies of recent key articles

(including health technology assessments ${ }^{4}{ }^{30}$ ) for these factors. This review was restricted to:

1. trials with more than 1,000 participants receiving tissue-plasminogen activator (tPA) for AMI, and

2. meta-analyses of pre-hospital thrombolysis trials for AMI.

Large trials with at least 1,000 participants were selected as this reduced the risk of having small numbers when data were disaggregated into different time points ${ }^{31}$. Studies of interventions/treatments not available in Australia were excluded (e.g. anistreplase ${ }^{32}$ ). For trials that compared tPA with another thrombolytic (such as streptokinase) or augmented tPA (e.g. tenecteplase plus enoxaparin in the ASSENT 3 trial $^{16}$ ), data for the tPA arm only were extracted. 
Several studies were identified that met the inclusion criteria, including GUSTO1, GUSTO3, GUSTO5, ASSENT2, ASSENT3, COBALT, INJECT, and InTime2 ${ }^{13-19}$. The results of the meta-analyses identified ${ }^{82} 23$ were dominated by the inclusion of anistreplase ${ }^{32}$; the efficacy profile of anistreplase is moderately different to the later tPA's and this is unavailable in Australia. However, one of these meta-analyses reported 30day mortality for $\mathrm{tPA}^{23}$; mortality was $5.5 \%, 5.6 \%$ and $12.4 \%$ for tPA given within 2 hours, 2-4 hours, and greater than 4 hours respectively. Because of the relatively small numbers receiving tPA in that study, the large studies were used primarily.

Data on the time-to-needle and 30-day mortality were extracted from the selected studies. The data were then analysed using weighted least squares (WLS) and coefficients for time to event calculated (Figure 2). Results of the analysis show the absolute risk - that is, if thrombolysis was administered at time $t=0$ minutes, the expected 30-day mortality is $4.2 \%$ (Table 2 ) and for every hour delay in treatment, mortality increases by $0.82 \%$.

FIGURE 2 GOES ABOUT HERE

TABLE 2 GOES ABOUT HERE

In the large tPA trials, mortality was often the only outcome that was reported with differential timing of treatment. In some of these trials, HF was reported but was not defined. Therefore, the mean TTN was used to estimate a linear equation for treatment delay on HF rates using results for the appropriate groups from the GUSTO1, GUSTO3, GUSTO5, ASSENT2, ASSENT3, INJECT, and InTime2 trials ${ }^{13} 14$ 16-19 33 . The equation was constrained to have a non-negative intercept. The slope of the WLS was 0.0368 
per hour delay (i.e. HF occurs at a rate of $3.7 \%$ per hour of treatment delay). The estimated effect is much smaller than that found in a pre-hospital vs in hospital thrombolysis study on HF (NYHA $\geq$ class II) using streptokinase ${ }^{24}$. That study reported mean HF rates of $1.5 \%$ and $16.0 \%$ for thrombolysis at 72 minutes and 120 minutes respectively ${ }^{24}$.

Parameters for other events are assumed to be independent of time-to-needle. The rates of other events, including the rate of anaphylaxis from tPA, death from anaphylaxis, the incidence of major bleeds, death from a major bleed, major disabling stroke, death from a major stroke, and subsequent interventional procedures were drawn directly from the literature (Table 3). Many of these probabilities were drawn from the ASSENT trials ${ }^{1}{ }^{16}$, ISIS- $3^{33}$, Kellet and Clarke ${ }^{26}$, Zijlstra et al. for subsequent procedures $^{22}$, and a health technology report ${ }^{4}$. There is no evidence to show that earlier treatment reduces interventional procedures after 30-days. Therefore, the rate of these procedures (and any complications and associated outcomes) is the same in both prehospital and in-hospital treatment survivors. Age-specific mortality for Australia was used to populate the model for those who survive the initial $\mathrm{AMI}^{34}$.

\section{TABLE 3 GOES ABOUT HERE}

Additional assumptions used

- The probability of dying from an AMI is highly age-dependent, ranging from $2.5 \%$ at 50 years of age to $25 \%$ at 80 years of age ${ }^{26}$. An initial age of 62 years is used. 
- Patients with a major disabling stroke do not recover and are equally likely to die regardless of initial treatment strategy. The excess mortality rate for patients surviving a stroke is $9.0 \%{ }^{26}$.

- The probability of dying from a major bleed (defined as requiring a blood transfusion) is constant regardless of the initial treatment strategy. The probability of dying from a major bleed increases linearly with age from $5 \%$ to $50 \%{ }^{26}$.

- Once patients have experienced HF, they are equally likely to die regardless of initial treatment strategy. HF results in an excess mortality rate of $8 \%{ }^{26}$.

- Surgical revascularisation is contraindicated for patients who experience either stroke or HF.

- No further revascularisation procedures are performed once a patient has undergone CABG.

- HF patients (without stroke) may have resynchronisation therapy with an implanted device (e.g. single or dual chamber pacemaker, implantable cardioverter defibrillator). An annual rate of $16.6 \%$ for these patients is used based on Queensland Health data ${ }^{35}$.

\section{Health-Related Quality of Life}

Previous studies reported that patients attributed a QALY weight of approximately 0.9 to their state of health after surviving AMI (Table 4). The value assigned to HF $(0.622)$ is the mean of the time-trade-off scores assigned to each of the four New York Heart Association (NYHA) HF classifications ${ }^{36}$. Similar utility weights are obtained using means weighted by NYHA II - IV prevalence (e.g. 0.63 is obtained using UK prevalence rates $)^{37}$ The value assigned to major disabling stroke $(0.36)$ is the 
median taken from a comprehensive review of all quality-of-life estimates for stroke appearing in peer-reviewed journals between 1985 and $2000^{38}$. This falls roughly halfway between the EQ-5D figures reported by Post et al. for minor (0.64) and major (0.04) stroke ${ }^{39}$.

\section{TABLE 4 GOES ABOUT HERE}

\section{Cost data}

All costs are in 2004 Australian dollars (A\$1 U US\$0.80). Hospital costs were assessed for Queensland Public Hospitals using the Australian Revised Diagnostic Related Groups (AR-DRG) version $5^{35}$ (Table 5). The cost of an AMI admission was adjusted to exclude the cost of thrombolytic medications (typically included with inpatient costs). Marginal costs were used for inpatient events that occur during the index AMI admission (i.e. anaphylaxis, major bleeds, death, $\mathrm{PCl}$ within 30 days, stroke and stroke deaths); this approach was necessary to avoid double-counting of AR-DRG costs because of the sequence-of-events structure of the model. These marginal costs (and the associated length of stay) were calculated as the difference between inpatient costs for $\mathrm{AMI}$ and the higher cost event (e.g. the mean cost for a $\mathrm{PCl}$ within 30 days of admission for $\mathrm{AMI}$ is $\mathrm{A} \$ 8218$ and the cost for $\mathrm{AMI}$ is $\mathrm{A} \$ 3638$; thus, the marginal cost of $\mathrm{PCl}$ is $\mathrm{A} \$ 4580)$. This approach is likely to understate the true cost as the cost for $\mathrm{AMI}$ is absorbed into the higher cost event.

\section{TABLE 5 GOES ABOUT HERE}


Ongoing prescription costs are incurred by AMI survivors. These costs include the cost of antihypertensives, ACE inhibitors, statins, nitrates, and so forth. Those with HF incur higher ongoing prescription costs for medications such as diuretics, beta blockers, and calcium channel inhibitors. We assumed an annual cost of $A \$ 1200$ for those with $\mathrm{AMI}$ uncomplicated $(\mathrm{A} \$ 1200$ is sufficient to purchase approximately four generic medications per year (e.g. Glyceryl trinitrate 100mcg $A \$ 11.27 / 100$; labetalol 100mg $A \$ 14.63 / 100$; simvastatin 40mg $A \$ 54.65 / 30$; aspirin 100mg $A \$ 7.11 / 112)^{40}$, and $50 \%$ higher cost for those with HF (e.g. Hydrochlorothiazide with Amiloride $\mathrm{HCl} 50 \mathrm{mg}+$ $5 \mathrm{mg} A \$ 11.64 / 100 ;$ Captopril 25mg A $\$ 24.37 / 90)^{40}$. GP and cardiology out-patient visits costs are expected to be incurred every three months; the cost for residential care for those with a major stroke was estimated as the maximum daily Medicare fee for residential care.

\section{Estimation of the model}

The model was estimated using Monte Carlo microsimulations. Microsimulations (first-order trials) use random number sequences to send one individual at a time through a randomly selected pathway in the model. The chance of a patient progressing down one pathway opposed to another is governed by the probability value at each node in the model. Tracker variables are used to count events. Averaging the results of a large number of individual trials approximates the expected value. Events, health outcomes (QALYs and life years) and costs are averaged for the microsimulations.

For the health state transition process for the period following the initial 30-days, monthly cycles are used. Rates of events that occur after the initial 30-day period were converted to transition probabilities (e.g. annual rates of $\mathrm{PCl}$ and death were converted 
to monthly probabilities) ${ }^{29}$. Half-cycle corrections were applied to the initial and final cycles in the process. The model was run with time horizons of 30 -days, 1 -year, 10years and rest of life.

\section{Discounting}

All costs and health outcomes were discounted at a rate of $5 \%$ following the Pharmaceutical Benefits Advisory Committee guidelines ${ }^{41}$.

\section{Sensitivity analysis}

One-way sensitivity analyses are undertaken around the key parameters with the greatest uncertainty. The purpose of the one-way sensitivity analysis is to estimate the robustness of the results in the face of changes in values of a single parameter. The rest of life horizon is used for the sensitivity analysis.

\section{RESULTS}

Estimated events occurring in the initial 30-days for a simulated cohort of 10,000 STEMI patients are shown in Table 6. Earlier administration of thrombolytics by Paramedics would avert 65 deaths per 10,000 cases at 30 days. This is a $10.5 \%$ reduction in 30-day mortality. The main component of these deaths was MI deaths; the relative reduction in $\mathrm{Ml}$ deaths was $13.3 \%$. Other events with noticeable differences between groups included the slightly higher numbers of $\mathrm{CABG}$ and PCl's performed within 30 -days, the $40.3 \%$ lower incidence of HF, and the slightly longer duration of hospital in-patient stays under Paramedic Practice compared with Current Practice. 
Overall, the costs of paramedic practice are $\$ 105$ greater than current practice due additional use of healthcare resources from increased survival.

\section{TABLE 6 GOES ABOUT HERE}

The subsequent events, procedures, health outcomes and costs estimated to occur over the first year, 10-years and rest of life horizons for the simulated cohort of 10,000 are shown in Table 7 . The rate (per 10,000 cases) of CABG and PCl continues to be slightly higher under Paramedic Practice over all three time horizons largely due to better survival of the initial event allowing the opportunity for revascularisation procedures later on. However, resynchronisation therapies are greater under Current Practice; this is due to the greater HF rates requiring pacemakers and ICDs. There is a noticeable increase in procedures as the time horizon is increased, as expected.

\section{TABLE 7 GOES ABOUT HERE}

The number of PCl's was also explored further. Of the 10,000 hypothetical patients, 4494 in the Paramedic Practice and 4344 in the Current Practice group received a $\mathrm{PCl} ; 3354(74.6 \%)$ and $3236(74.5 \%)$ received one $\mathrm{PCl}$ only and 6 in both

groups $(0.014 \%$ and $0.013 \%)$ received a total of $5 \mathrm{PCls}$ over the rest of life. The increased survival and increased revascularisation rate contributed to the slightly increased total days stay in hospital over the rest of life.

Because there are slightly fewer deaths in the first 30-days under Paramedic Practice, the number of expected life-years is slightly greater over the three time horizons. Quality-adjusting life-years gave a similar picture and incorporates the gain in 
quality of life from a lower rate of HF; likewise, costs are also slightly greater under Paramedic Practice. These additional costs are due to greater survival in the early stages which incurs additional consumption of healthcare resources at a later stage. On average, STEMI patients can expect to live 0.10 years longer, gain 0.13 QALYs and incur an additional $\$ 343$ to the health system over remaining life under Paramedic Practice. Thus, the incremental cost-effectiveness ratios (ICERs) are $\$ 3428$ per life-year gained and \$2601 per QALY gained over the rest of life.

To depict the distribution of the ICERs from the simulations, a scatterplot (with a $95 \%$ confidence ellipse) on the cost-effectiveness plane is used (Figure 3 ). The vast majority of the simulations $(98.1 \%)$ resulted in higher costs and better health outcomes from Paramedic Practice, and in 1.5\%, Paramedic Practice resulted in lower costs and worse health outcomes. Paramedic Practice was dominated in $0.2 \%$ of the simulations with higher costs and worse health outcomes and dominant in $0.2 \%$ of the simulations.

FIGURE 3 GOES ABOUT HERE

Sensitivity analysis results

The one-way sensitivity analysis is presented as a tornado diagram (Figure 4) using the rest of life horizon. The QALY weight for good health, discount rates for health benefits and costs, the cost of resynchronisation therapy, and cost of medications (with and without $\mathrm{HF}$ ) were the six variables that had the greatest effect on the ICER. A lower QALY weight for good health resulted in higher ICER as fewer QALYs are gained.

FIGURE 4 GOES ABOUT HERE 
A higher discount rate reduced health benefits and costs that occur in the future, and vice versa. The ICER increased to \$3797/QALY when health outcomes were discounted at $10 \%$ and the decreased to $\$ 1801 /$ QALY when health outcomes were not discounted. Results were less sensitive to changes in the discount rate for costs. When both costs and health outcomes were discounted at 0\%, the ICER was $\$ 2447$ per QALY and when both were discounted at $10 \%$ the ICER was $\$ 3148$.

The lower cost estimates for ongoing medications (both with and without HF) had a large effect on incremental costs and a lesser effect on the ICERs. The costs of resynchronisation therapy had a relatively large effect on the ICER; if the upper cost for resynchronisation therapy is used, (i.e. an ICD with complications or comorbidities), the ICER decreased by $65 \%$. Other than these factors, changes in all other costs had relatively little effect.

Decreasing the 30-day probability of death reduced the incremental costs and QALYs. However, with a lower death rate, absolute costs and the number of QALYs increased slightly, and vice versa. Similar effects were seen with the time-dependent parameter for developing HF.

Changing the probabilities of subsequent interventions by $+/-20 \%$ made less than $+/-5 \%$ difference on the ICERs. Changes in these parameters were largely confined to affecting costs rather than QALYs. The long-term CABG probability was the most sensitive parameter in this group. 
The TTN had the smallest effect on the ICER. However, variation of the TTN had large, and proportionate, effects on both the incremental costs and the incremental QALYs. For example, reducing the Paramedic TTN increased both the incremental QALYs and incremental costs by a similar magnitude (Table 8). The additional costs and QALYs are due to increased survival. When the time to paramedic treatment is close to that of hospital treatment (120 minutes vs 126 minutes), both the incremental costs and incremental health benefits are small.

\section{TABLE 8 GOES ABOUT HERE}

\section{DISCUSSION}

This study estimated the cost-effectiveness of introducing earlier administration of thrombolytics by paramedics in the field compared with the Current Practice of administering thrombolytics only in hospital. Results showed that Paramedic Practice could reduce 30 -day mortality by $10.5 \%$, and increase the median life expectancy of a 62-year old by two months on average. Paramedic Practice could make a large difference to the incidence of HF reducing this by $40 \%$. This reduction in HF leads to fewer demands on health care resources (e.g. reduced use pharmaceuticals and lower rates of resynchronisation therapies). However, additional survival led to increased future health care resource use and costs (including pharmaceuticals and GP visits).

The most important factor was the TTN - the greater the difference between Current Practice times and paramedic times, the greater the health benefits; however, with increased survival, future costs also increased proportionately. Thus, there was little change in the ICER from variation in the TTN (i.e. both the numerator (costs) and 
denominator (health outcomes, survival, QALYs) were increased by the same factor). In addition, age had relatively little effect on the cost per QALY; thus, there is no argument to limit thrombolysis to above or below an age threshold.

Overall, STEMI patients can expect to gain 0.13 QALYs and incur an additional $\$ 343$ to the health system over 30 years under Paramedic Practice. Thus, there are additional health benefits but additional costs. The incremental cost-effectiveness ratios (ICERs) were \$3428 per life-year gained and \$2601 per QALY gained. Sensitivity analysis showed that the ICERs varied over a very small range when the input parameters were changed by $+/-20 \%$. Thus, the ICERs are relatively stable. These ICERs are low compared with the ICERs for new pharmaceuticals recommended by the PBAC for listing on the PBS where the implicit upper acceptable limit is around $\$ 70,000$ per life-year gained ${ }^{42}$. Thus, the proposed administration of thrombolysis by paramedics is good value for money.

These results are more favourable than a similar study undertaken in the UK which estimated the cost-effectiveness of combined reductions in ambulance response times (from 10.4 minutes to 8 minutes) and arrival at hospital to thrombolysis (in which $75 \%$ of patients received thrombolysis within 80 minutes compared to thrombolysis within 30 minutes $)^{43}$. The on-scene and transport to hospital times were not reported in that study ${ }^{43}$; however in our study these times accounted for approximately 40 minutes in which paramedics could administer thrombolytics on average 17 minutes before arrival at hospital. Interestingly, the modelled reduction in time to thrombolysis was 52 minutes in both studies, but the points of intervention were quite different. The UK study modelled a general population of 500,000 whereas we modelled only those with a STEMI event. Cases of cardiogenic chest pain that were not STEMI would be treated in 
the usual manner (i.e. not given thrombolysis by paramedics) and therefore were not modelled in our study. However, the UK study did not explicitly consider long-term costs and effects of heart failure or stroke; these factors were a key determinant of the costeffectiveness in our study which, in addition to the different populations modelled, we suspect are the main cause of the difference in results.

There are potentially large costs to establish a service where paramedics administer thrombolysis. These costs include stocking ambulances with thrombolytics and the training of staff to administer thrombolytics. However, these costs occur early in the establishment phase and may be considered as an initial investment to provide long term future health gains. In addition, there may be some increased wastage due to a limited shelf-life of the thrombolytics (2-3 years) but conversely discounts for bulk purchases may be obtained. Because these factors are not directly related to the economic efficiency of providing the service, but are related to establishing a service and the management of change from one practice to another, they should all be considered in a budget impact assessment rather than a cost-effectiveness analysis.

There are some limitations of the analysis, most of which relate to data availability and quality. The model includes the most clinically and economically significant consequences associated with STEMI. Data on the incidence and transition probabilities were obtained largely from literature reporting multi-centre international trials and local (Queensland) databases on TTN. Most studies failed to disaggregate secondary outcomes by TTN; HF and other secondary outcomes may have substantial effects on quality of life (and hence QALYs). Therefore, the effect of TTN on rates of events and other secondary outcomes is the greatest uncertainty and limitation of this study. In addition, the utility weights for subsequent health states, including heart failure 
and stroke, were based on mean severity; a different distribution of severity such as a greater proportion of NYHA III or IV would produce a lower heart failure utility weight and thus increase the benefits of earlier TTN. However, sensitivity analysis showed that the ICERs were relatively robust to variation in all parameter values.

The thrombolytic modelled in this study was tenecteplase. However, there is a range of thrombolytics used in current hospital practice including alteplase, reteplase, and in some case streptokinase. Other thrombolytics have been superseded (e.g. anistreplase) and others are relatively new to the market (e.g. lanoteplase $)^{19}$. Moreover, the use of $\mathrm{PCl}$ as the primary treatment for thrombolysis is becoming more common ${ }^{44}$ and is the recommended guideline under certain conditions (e.g. where $\mathrm{PCl}$ can be commenced within 90 minutes of presentation ${ }^{5}{ }^{45}$ ). Paramedics administering thrombolytics will reduce the chance for primary $\mathrm{PCl}$ to subsequently occur but the time to primary $\mathrm{PCl}$ is longer than thrombolysis leading to potentially greater health benefits from paramedic administered thrombolysis ${ }^{23}$. Based on cost-effectiveness grounds, a UK study has shown that primary $\mathrm{PCI}$ is more favourable compared with thrombolysis; however, there are substantial infrastructure difficulties with many hospitals not equipped for angioplasty and even fewer with the capacity to be able to administer primary $\mathrm{PCl}$ within the recommended times ${ }^{30}$. Therefore, paramedic administered thrombolysis is a feasible and viable approach to improve health outcomes.

This study has established that a reduction in the time-to-needle is the key to the economic feasibility of thrombolytics administered by paramedics. Administering thrombolysis by paramedics requires correctly identifying STEMI in patients with chest pain. This present study has assumed that all STEMI patients who would be thrombolysed are correctly identified based on preliminary work by Murray et al. ${ }^{11}$ An 
intensive training programme for all paramedics in 12-lead ECG acquisition and interpretation has been implemented in Queensland ${ }^{46}$; ICPs have also had specific face-to-face education on fibrinolysis and carry a checklist and decision matrix for administering tenecteplase. Errors in diagnosis may cause the cost-effectiveness estimates to change. However, practice guidelines do not permit the use of thrombolytics where there is any doubt regarding the diagnosis.

Paramedics undertaking to diagnose and treat patients with thrombolysis without having a clinician verify the diagnosis could result in some cases of litigation, and some may argue that only specialist cardiologists can provide the best evidence based care ${ }^{47}$. The definition of AMI, and hence strict diagnostic criteria for STEMI, is complex with a spectrum into unstable angina with ST elevation being an important issue ${ }^{45} 4849$. Alternative approaches to confirm a diagnosis which enable earlier time-to-needle (with paramedic administered thrombolytics) have been instigated in some countries. For example, through real-time telemedicine in Norway, the ECG is transmitted to an emergency department at a base hospital for the diagnosis of STEMI to be confirmed by a clinician and treatment instigated ${ }^{50}$. However, Intensive Care Paramedics (ICPs) in Queensland are among the most highly trained paramedics in the world. All have a mandatory minimum of six years clinical practice prior to enrolment in the post-graduate Diploma of Intensive Care practice. Practitioners at this level have an expanded scope of practice and access to a wider range of drug therapies (e.g. ketamine, midazolam). In addition, work currently in progress demonstrates clearly that appropriately trained ICPS are able to correctly identify patients appropriate for lysis based on interpretation of 12lead ECG's at least as frequently as two cardiologists blinded to the paramedic decision to lyse or not. In addition, where there is any uncertainty around a diagnosis of STEMI by the paramedics, the patient is transported to hospital for diagnosis in the emergency 
department; this occurs with current practice and is expected to continue for a minority of patients whose diagnosis is more challenging. That is, there is a small chance that some STEMI patients will not be given thrombolysis as early as possible due to this uncertainty with an increased relative risk of poorer outcomes (i.e. as occurs with current practice); conversely, there is a risk that non-STEMI patients may be given thrombolysis resulting in potentially unnecessary bleeds.

These results may be generalised not only to other States in Australia, but internationally. The condition for applicability of these results requires similarly trained paramedics including competence in acquisition and interpretation of 12-lead ECG's and the administration of thrombolytics. In addition, for similar results response and transportation times should also be similar to those in this study.

\section{CONCLUSION}

Earlier administration of thrombolytics by paramedics could reduce 30-day mortality by $10.5 \%$ and the incidence of heart failure by $40 \%$. STEMI patients can, on average, expect to live 0.10 years longer, gain 0.13 QALYs and incur an additional $\$ 343$ to the health system over remaining life from paramedic-administered thrombolysis. The incremental cost-effectiveness ratios (ICERs) are \$3428 per life-year gained and \$2601 per QALY gained over the rest of life. This healthcare investment is good value for money.

Compared with Australia, the health benefits and cost-savings may be substantially greater in other countries where the difference in time between pre-hospital 
and in-hospital thrombolysis is greater than the 52-minute difference noted in Queensland. 


\section{Acknowledgments}

This study was funded by the Queensland Ambulance Service, Australia. The study was undertaken on an exploratory basis to assess whether thrombolytics administered by paramedics would be financially viable. There were no conflicts of interest in this costeffectiveness and budget impact assessment. The authors thank Intensive Care Paramedics Brett Rogers and John Murray for their assistance in obtaining data and providing comments on an earlier draft of this paper. In addition, we are grateful to the reviewers who provided valuable and constructive comments which have help to substantially improve this article. 


\section{REFERENCES}

1. Assessment of the Safety and Efficacy of a New Thrombolytic (ASSENT)-2 Investigators. Single bolus tenecteplase compared with front-loaded alteplase in acute myocardial infarction: the ASSENT-2 double-blind randomised trial. The Lancet 1999;354:716-722.

2. Randomised, double-blind comparison of reteplase double-bolus administration with streptokinase in acute myocardial infarction (INJECT): trial to investigate equivalence. International Joint Efficacy Comparison of Thrombolytics. Lancet 1995;346(8971):329-36.

3. Dundar Y, Hill R, Dickson R, Walley T. Comparative efficacy of thrombolytics in acute myocardial infarction: a systematic review.[see comment]. Qjm 2003;96(2):103-13.

4. Boland A, Dundar Y, Bagust A, Haycox A, Hill R, Mujica Mota R, et al. Early thrombolysis for the treatment of acute myocardial infarction: a systematic review and economic evaluation. Health Technol Assess 2003;7(15).

5. National Heart Foundation of Australia and Cardiac Society of Australia and New Zealand. Guidelines for the management of acute coronary syndromes 2006. MJA 2006;184(Suppl 8):S1-S32.

6. Scottish Intercollegiate Guidelines Network. Acute Coronary Syndromes - A National Clinical Guideline. Edinburgh: Scottish Intercollegiate Guidelines Network, 2007.

7. Brouwer MA, Martin JS, Maynard C, Wirkus M, Litwin PE, Verheugt FW, et al. Influence of early prehospital thrombolysis on mortality and event-free survival (the Myocardial Infarction Triage and Intervention [MITI] Randomized Trial). MITI Project Investigators. Am J Cardiol 1996;78(5):497-502.

8. Morrison LJ, Verbeek PR, McDonald AC, Sawadsky BV, Cook DJ. Mortality and prehospital thrombolysis for acute myocardial infarction: A meta-analysis. JAMA 2000;283(20):2686-92.

9. Rawles JM. Quantification of the benefit of earlier thrombolytic therapy: five-year results of the Grampian Region Early Anistreplase Trial (GREAT). J Am Coll Cardiol 1997;30(5):1181-6.

10. Department of Health, editor. Coronary Heart Disease: National Service Frameworks: Department of Health, 2000.

11. Murray J, Rogers B, et al. Early identification of patients for thrombolysis by intensive care paramedics - A pilot study. Brisbane: Queensland Ambulance Service, 2004.

12. Criteria Committee of the American Heart Association (New York City Affiliate). Nomenclature and criteria for diagnosis of disease of the heart and great vessels 9th ed. Boston, MA: Little Brown \& Co., 1994.

13. The Gusto Investigators. An international randomized trial comparing four thrombolytic strategies for acute myocardial infarction. $N$ Engl $J$ Med 1993;329(10):673-682. 
14. GUSTO III. A comparison of reteplase with alteplase for acute myocardial infarction. The Global Use of Strategies to Open Occluded Coronary Arteries (GUSTO III) Investigators. New England journal of medicine 1997;337(16):1118-23.

15. The GUSTO V Investigators. Reperfusion therapy for acute myocardial infarction with fibrinolytic therapy or combination reduced fibrinolytic therapy and platelet glycoproyein IIb/IIIa inhibition: the GUSTO V randomised trial. Lancet 2001;357:1905-1914.

16. The Assessment of the Safety and Efficacy of a New Thrombolytic Regimen (ASSENT)-3 Investigators. Efficacy and safety of tenecteplase in combination with enoxaparin, abciximab, or unfractionated heparin: the ASSENT-3 randomised trial in acute myocardial infarction. The Lancet 2001;358:605-613.

17. Van de Werf F, Adgey A, Agnelli G, Aylward PeaTCI, . A comparison of continuous infusion of alteplase with double-bolus administration for acute myocardial infarction. New England Journal of Medicine 1997;337(16):1124-1130.

18. International Joint Efficacy Comparison of Thrombolytics. Randomised, double-blind comparison of reteplase double-bolus administeration with streptokinase in acute myocardial infarction (INJECT): trial to investigate equivalence. The Lancet 1995;346:329-336.

19. The InTIME-II Investigators. Intravenous NPA for the treatment of infarcting myocardium early. InTIME-II, a double-blind comparison of single-bolus lanoteplase vs accelerated alteplase for the treatment of patients with acute myocardial infarction. Eur Heart J 2000;21(24):2005-2013.

20. Boersma E, Maas AC, Deckers JW, Simoons ML. Early thrombolytic treatment in acute myocardial infarction: reappraisal of the golden hour.[see comment]. Lancet 1996;348(9030):771-5.

21. Weaver WD, Simes RJ, Betriu A, Grines CL, Zijlstra F, Garcia E, et al. Comparison of primary coronary angioplasty and intravenous thrombolytic therapy for acute myocardial infarction: a quantitative review.[see comment][erratum appears in JAMA 1998 Jun 17;279(23):1876]. JAMA 1997;278(23):2093-8.

22. Zijlstra F, Hoorntje JC, de Boer MJ, Reiffers S, Miedema K, Ottervanger JP, et al. Long-term benefit of primary angioplasty as compared with thrombolytic therapy for acute myocardial infarction. New England journal of medicine 1999;341(19):1413-9.

23. Zijlstra F, Patel A, Jones M, Grines CL, Ellis S, Garcia E, et al. Clinical characteristics and outcome of patients with early $(<2 \mathrm{~h})$, intermediate $(2-4 \mathrm{~h})$ and late $(>4 \mathrm{~h})$ presentation treated by primary coronary angioplasty or thrombolytic therapy for acute myocardial infarction. Eur Heart J 2002;23(7):550-7.

24. Weiss AT, Leitersdorf I, Gotsman MS, Zahger D, Sapoznikov D, Rozenman Y, et al. Prevention of congestive heart failure by early, prehospital thrombolysis in acute myocardial infarction: a long-term follow-up study. Int J Cardiol 1998;65 Suppl 1:S43-8.

25. Weaver WD, Cerqueira M, Hallstrom AP, Litwin PE, Martin JS, Kudenchuk PJ, et al. Prehospital-initiated vs hospital-initiated thrombolytic therapy. The Myocardial Infarction Triage and Intervention Trial. JAMA 1993;270(10):1211-6.

26. Kellett J, Clarke J. Comparison of "accelerated" tissue plasminogen activator with streptokinase for treatment of suspected myocardial infarction. Medical decision 
making an international journal of the Society for Medical Decision Making 1995;15(4):297-310.

27. Kellett J. Cost-effectiveness of accelerated tissue plasminogen activator for acute myocardial infarction. British Journal of Medical Economics 1996;10:341-359.

28. TreeAge Pro [program]. Williamstown, MA: TreeAge Software Inc., 2006.

29. Sonnenberg FA, Beck JR. Markov models in medical decision making: a practical guide. Medical Decision Making 1993;13 322-338.

30. Hartwell D, Colquitt J, Loveman E, Clegg AJ, Brodin H, Waugh N, et al. Clinical effectiveness and cost-effectiveness of immediate angioplasty for acute myocardial infarction: systematic review and economic evaluation. Health Technol Assess 2005;9(17):1-114.

31. Appleby P, Baigent C, Collins R, Flather M, et al. Indications for fibrinolytic therapy in suspected acute myocardial infarction: collaborative overview of early mortality and major morbidity results from all randomised trials of more than 1000 patients. Lancet 1994;343(8893):311-322.

32. The European Myocardial Infarction Project Group. Prehospital thrombolytic therapy in patients with suspected acute myocardial infarction. $N$ Engl $J$ Med 1993;329(6):383-389.

33. ISIS-3 Collaborative Group. ISIS-3: a randomised comparison of streptokinase vs tissue plasminogen activator vs anistreplase and of aspirin plus heparin vs aspirin alone among 41,299 cases of suspected acute myocardial infarction. Lancet 1992;339(8796):753-70.

34. Australian Institute of Health and Welfare (AIHW). GRIM (General Record of Incidence of Mortality) Books. Canberra: AIHW, 2005.

35. Australian Institute of Health and Welfare (AIHW). Cost Weights for AR-DRG Version 5.0, Round 8 (2003-04) - Queensland National Hospital Cost Data Collection. Canberra: AIHW, 2005.

36. Kirsch J, McGuire A. Establishing health state valuations for disease specific states: an example from heart disease. Health economics 2000;9(2):149-58.

37. Hobbs FDR, Kenkre JE, Roalfe AK, Davis RC, Hare R, Davies MK. Impact of heart failure and left ventricular systolic dysfunction on quality of life. A crosssectional study comparing common chronic cardiac and medical disorders and a representative adult population. Eur Heart J 2002;23(23):1867-1876.

38. Tengs TO, Yu M, Luistro E. Health-related quality of life after stroke a comprehensive review. Stroke a journal of cerebral circulation 2001;32(4):96472.

39. Post PN, Stiggelbout AM, Wakker PP. The utility of health states after stroke: a systematic review of the literature. Stroke a journal of cerebral circulation 2001;32(6):1425-9.

40. Department of Health and Ageing. Pharmaceutical Benefit Schedule, April 2008. Canberra: DoHA, Australian Government, 2008.

41. Commonwealth Department of Health and Ageing. Guidelines for the Pharmaceutical Industry on Preparation of Submissions to the Pharmaceutical Benefits Advisory Committee. Canberra: Publications Production Unit, 2002. 
42. George B, Harris A, Mitchell A. Cost-effectiveness analysis and the consistency of decision making: evidence from pharmaceutical reimbursement in australia (1991 to 1996). Pharmacoeconomics 2001;19(11):1103-09.

43. Chase D, Roderick P, Cooper K, Davies R, Quinn T, Raftery J. Using simulation to estimate the cost effectiveness of improving ambulance and thrombolysis response times after myocardial infarction. Emerg Med J 2006;23(1):67-72.

44. British Cardiovascular Intervention Society. Audit Returns of Interventional Procedures 2004. London: BCIS, 2004.

45. Antman EM, Anbe DT, Armstrong PW, Bates ER, Green LA, Hand M, et al. ACC/AHA Guidelines for the Management of Patients With ST-Elevation Myocardial Infarction--Executive Summary: A Report of the American College of Cardiology/American Heart Association Task Force on Practice Guidelines (Writing Committee to Revise the 1999 Guidelines for the Management of Patients With Acute Myocardial Infarction). Journal of the American College of Cardiology 2004;44(3):671-719.

46. Queensland Ambulance Service. The 12-lead ECG in Paramedic Practice. Brisbane: Queensland Ambulance Service, Department of Emergency Services, 2007.

47. Birkhead JS, Weston C, Lowe D, National Audit of Myocardial Infarction Project Steering G. Impact of specialty of admitting physician and type of hospital on care and outcome for myocardial infarction in England and Wales during 2004-5: observational study. BMJ 2006;332(7553):1306-1311.

48. Bassand J-P, Hamm C. Guidelines for the diagnosis and treatment of non-ST-segment elevation acute coronary syndromes: The Task Force for the Diagnosis and Treatment of Non-ST-Segment Elevation Acute Coronary Syndromes of the European Society of Cardiology: reply. Eur Heart J 2008;29(2):278-279.

49. Rapezzi C, Biagini E, Branzi A. Guidelines for the diagnosis and treatment of nonST-segment elevation acute coronary syndromes: The Task Force for the Diagnosis and Treatment of Non-ST-Segment Elevation Acute Coronary Syndromes of the European Society of Cardiology. Eur Heart J 2008;29(2):277a-278.

50. Ljosland M, Weydahl PG, Stumberg S. Prehospital ECG reduces the delay of thrombolysis in acute myocardial infarction. [In Norwegian]. Tidsskr Nor Laegeforen 2000;120(19):2247-49.

51. Betriu A, Phillips H, Ellis E. A clinical trial comparing primary coronary angioplasty with tissue plasminogen activator for acute myocardial infarction. New England Journal of Medicine 1997;336(23):1621-1629.

52. British Cardiovascular Intervention Society. Audit Returns of Interventional Procedures 2001. London: BCIS, 2001.

53. Department of Health and Ageing. Medicare Benefits Schedule Book. Canberra: DoHA, Australian Government, 2004.

54. Department of Health and Ageing. Revised residential care fees and charges: Schedule of Fees and Charges. Canberra: DoHA, Australian Government, 2004. 
Table 1: Paramedic response and treatments times based on 28,298 chest-pain calls in $2003^{11}$

\begin{tabular}{lcc}
\hline Event & $\begin{array}{c}\text { Paramedic } \\
\text { (mins) }\end{array}$ & $\begin{array}{c}\text { Hospital } \\
\text { (mins) }\end{array}$ \\
\hline Symptom onset to call & 43 & 43 \\
Response time & 10 & 10 \\
On-scene time & 21 & 21 \\
Transport to hospital ${ }^{*}$ & & 17 \\
Hospital handover & & 5 \\
Hospital diagnosis and treatment** & 30 \\
\hline Total time from symptom onset to lysis treatment: & 74 & 126 \\
\hline${ }^{*}$ Median = 12.9 mins; $90 \%<30$ mins, 95\% <45 mins & & \\
${ }^{* *}$ assumed & &
\end{tabular}


Table 2: Weighted least-squares estimates for 30-day mortality and HF

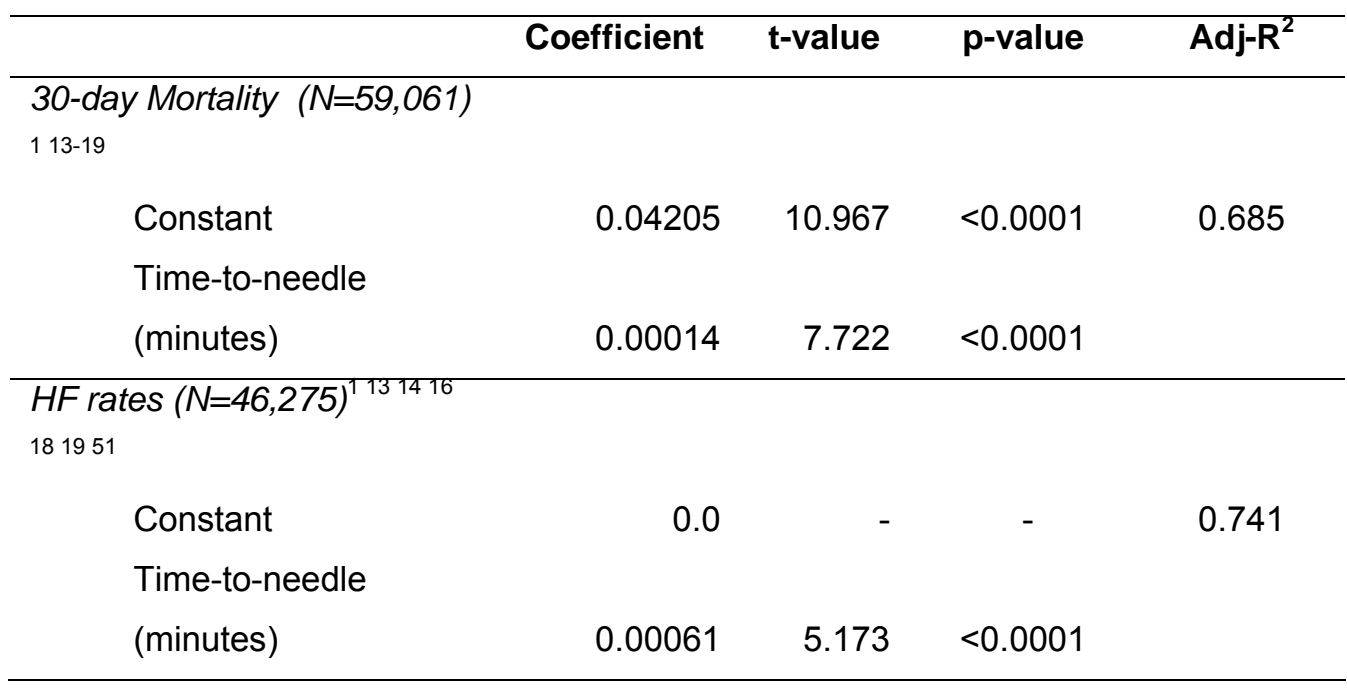


Table 3: Probability Data used in the model

\begin{tabular}{|c|c|c|}
\hline Event & Rates & Source \\
\hline \multirow[t]{2}{*}{ AMI 30-day mortality rate (\%) } & $4.20+$ & Table 2 \\
\hline & 0.82 / hour delay & \\
\hline Anaphylaxis (\%) & 0.2 & 1131427 \\
\hline Death from anaphylaxis (\%) & 5.0 & 27 \\
\hline Major bleed (\%) & 0.72 & 127 \\
\hline Death for those who have a major bleed (\%) & 12.76 & 27 \\
\hline Major disabling stroke $(\%)$ & 1.75 & 4 \\
\hline Death for those who have a major stroke (\%) & 49.1 & 33 \\
\hline Develop HF per hour treatment delay (\%) & 3.68 & Table 2 \\
\hline \multicolumn{3}{|l|}{ Subsequent procedures } \\
\hline $\mathrm{PCl} \leq 30$ days of thrombolytic therapy (\%) & 34.8 & 22 \\
\hline $\mathrm{PCl}>30$ days of thrombolytic therapy (annual \%) & 3.6 & 22 \\
\hline CABG $\leq 30$ days of thrombolytic therapy $(\%)$ & 8.0 & 22 \\
\hline CABG $>30$ days of thrombolytic therapy (annual \%) & 2.4 & 22 \\
\hline Resynchronisation therapy implant for HF (annual \%) & 16.6 & 35 \\
\hline \multicolumn{3}{|l|}{ Other parameters } \\
\hline CABG operative death (\%) & 4.0 & 52 \\
\hline $\mathrm{PCl}$ operative death $(\%)$ & 6.4 & 52 \\
\hline \multicolumn{3}{|l|}{ Excess mortality for MI survivors (annual \%) } \\
\hline - without HF & 2.2 & 27 \\
\hline - with HF & 8.0 & \\
\hline Excess mortality for stroke (annual \%) & 9.0 & 27 \\
\hline
\end{tabular}


Table 4: QALY weights associated with heart disease used in the model

\begin{tabular}{lc}
\hline Health State & QALY Weight \\
\hline Good health post AMI & 0.9 \\
Dead & 0.0 \\
Alive with HF & 0.622 \\
Alive with major disabling stroke & 0.36 \\
Alive with HF and stroke* & $0.622^{*} 0.36=0.224$ \\
${ }^{*}$ A multiplicative function (opposed to an additive) allows for the marginal loss of utility from having both HF \\
and stroke.
\end{tabular}


Table 5: Costs used in the model (all costs are in 2004 A\$)

\begin{tabular}{|c|c|c|c|}
\hline & Mean length & & \\
\hline Event & of stay (days) & Unit cost $(A \$)$ & Source \\
\hline Paramedic costs & & 300 & Assumption \\
\hline Tenecteplase & & 1300 & 11 \\
\hline \multicolumn{4}{|l|}{ Inpatient costs } \\
\hline AMI & 3.74 & 3638 & 35 \\
\hline Anaphylaxis * & 3.52 & 2542 & 35 \\
\hline Major bleed * & 3.97 & 4754 & 35 \\
\hline AMI Death * & 1.09 & 332 & 35 \\
\hline Stroke * & 8.26 & 5812 & 35 \\
\hline Stroke Death * & 1.49 & 1523 & 35 \\
\hline Heart failure * & 2.26 & 573 & 35 \\
\hline CABG & 9.49 & 18558 & 35 \\
\hline $\mathrm{PCl}<30$ days $*$ & 0.66 & 4580 & 35 \\
\hline $\mathrm{PCl}>30$ days & 2.28 & 5046 & 35 \\
\hline Resynchronisation therapy & 3.80 & 9515 & 35 \\
\hline Continuing costs & Frequency & Unit cost $(A \$)$ & Source \\
\hline Medication costs post AMI no HF & \multirow[t]{2}{*}{ Annual } & 1200 & Assumption \\
\hline Medication costs post AMI with HF & & 1800 & Assumption \\
\hline & Once every 3 & & \\
\hline \multirow[t]{2}{*}{ Outpatient visits } & months & 93.68 & \\
\hline & Once every 3 & & \\
\hline GP visits & months & 30.85 & 53 \\
\hline Major stroke ** & Annual ongoing & $10,453.5$ & 54 \\
\hline
\end{tabular}

* Indicates marginal cost

** Maximum fee for all respite residents 
Table 6: 30 -day events and costs for a simulated cohort of 10,000

\begin{tabular}{lrr}
\hline Event & \multicolumn{2}{c}{$\begin{array}{c}\text { Current } \\
\text { Practice }\end{array}$} \\
\hline Anaphylaxis & 13 & Practice \\
Anaphylaxis Deaths & 1 & 1 \\
Major bleeds & 67 & 67 \\
Death from major bleeds & 6 & 5 \\
CABG & 691 & 715 \\
Heart Failure & 647 & 386 \\
Mean in-patient stay (days) & 4.71 & 4.73 \\
MI Death & 488 & 423 \\
Operative deaths & 48 & 49 \\
PCl & 2994 & 3099 \\
Major stroke & 171 & 173 \\
Death from stroke & 78 & 78 \\
Total deaths & 621 & 556 \\
Costs & $\mathrm{A} \$ 816 \mathrm{~m}$ & $\mathrm{~A} \$ 826.5 \mathrm{~m}$ \\
\hline
\end{tabular}


Table 7: Health outcomes and events for a simulated cohort of 10,000 over 1-year, 10-years and rest of life

\begin{tabular}{|c|c|c|c|c|c|c|}
\hline & \multicolumn{2}{|c|}{1 year } & \multicolumn{2}{|c|}{10 years } & \multicolumn{2}{|c|}{ Rest of life } \\
\hline & $\begin{array}{l}\text { Current } \\
\text { Practice }\end{array}$ & $\begin{array}{c}\text { Paramedic } \\
\text { Practice }\end{array}$ & $\begin{array}{l}\text { Current } \\
\text { Practice }\end{array}$ & $\begin{array}{c}\text { Paramedic } \\
\text { Practice }\end{array}$ & $\begin{array}{l}\text { Current } \\
\text { Practice }\end{array}$ & $\begin{array}{c}\text { Paramedic } \\
\text { Practice }\end{array}$ \\
\hline CABG & 0.087 & 0.090 & 0.207 & 0.214 & 0.259 & 0.268 \\
\hline $\mathrm{PCl}$ & 0.339 & 0.350 & 0.501 & 0.519 & 0.573 & 0.592 \\
\hline Resynchronisation & 0.010 & 0.005 & 0.039 & 0.023 & 0.041 & 0.024 \\
\hline Operative death & 0.005 & 0.005 & 0.005 & 0.005 & 0.005 & 0.006 \\
\hline \multicolumn{7}{|l|}{ In patient stays } \\
\hline (days) & 5.053 & 5.088 & 7.482 & 7.593 & 8.740 & 8.894 \\
\hline Total deaths & 0.105 & 0.099 & 0.518 & 0.509 & 0.992 & 0.992 \\
\hline Life Years (LYs) & 0.894 & 0.900 & 5.721 & 5.792 & 7.373 & 7.473 \\
\hline QALYs & 0.784 & 0.795 & 5.040 & 5.139 & 6.510 & 6.642 \\
\hline Costs $(A \$)$ & 11,188 & 11,252 & 28,876 & 29,101 & 35,085 & 35,429 \\
\hline Incremental LYs & \multicolumn{2}{|c|}{0.005} & \multicolumn{2}{|c|}{0.071} & \multicolumn{2}{|c|}{0.100} \\
\hline Incremental QALYs & \multicolumn{2}{|c|}{0.011} & \multicolumn{2}{|c|}{0.099} & \multicolumn{2}{|c|}{0.132} \\
\hline Incremental costs & \multicolumn{2}{|c|}{64} & \multicolumn{2}{|c|}{225} & \multicolumn{2}{|c|}{343} \\
\hline ICER (LY) & \multicolumn{2}{|c|}{$\mathrm{A} \$ 11,850$} & \multicolumn{2}{|c|}{$\mathrm{A} \$ 3,174$} & \multicolumn{2}{|c|}{$A \$ 3,428$} \\
\hline ICER (QALY) & \multicolumn{2}{|c|}{$A \$ 5,579$} & \multicolumn{2}{|c|}{$\mathrm{A} \$ 2,262$} & \multicolumn{2}{|c|}{$A \$ 2,601$} \\
\hline
\end{tabular}


Table 8. Sensitivity analysis of time to needle

\begin{tabular}{lcccccc}
\hline & \multicolumn{3}{c}{ Low parameter value } & \multicolumn{3}{c}{ High parameter value } \\
& Inc. Cost & Inc. QALYs & ICER & Inc. Cost & Inc. QALYs & ICER \\
\hline Paramedic [74] $(50,120)$ & $46.4 \%$ & $46.5 \%$ & $-0.1 \%$ & $-88.6 \%$ & $-88.5 \%$ & $0.1 \%$ \\
Hospital [126] $(90,180)$ & $-69.2 \%$ & $-69.1 \%$ & $-0.1 \%$ & $103.1 \%$ & $102.9 \%$ & $0.2 \%$ \\
\hline
\end{tabular}




\section{Figure Captions}

Figure 1. Simplified depiction of the post-acute health state transition process

Figure 2. Time to needle and 30-day mortality

Figure 3. Scatterplot and 95\% confidence ellipse of the Incremental CostEffectiveness Ratios for the rest of life

Figure 4. One-way sensitivity analysis (rest of life horizon) 


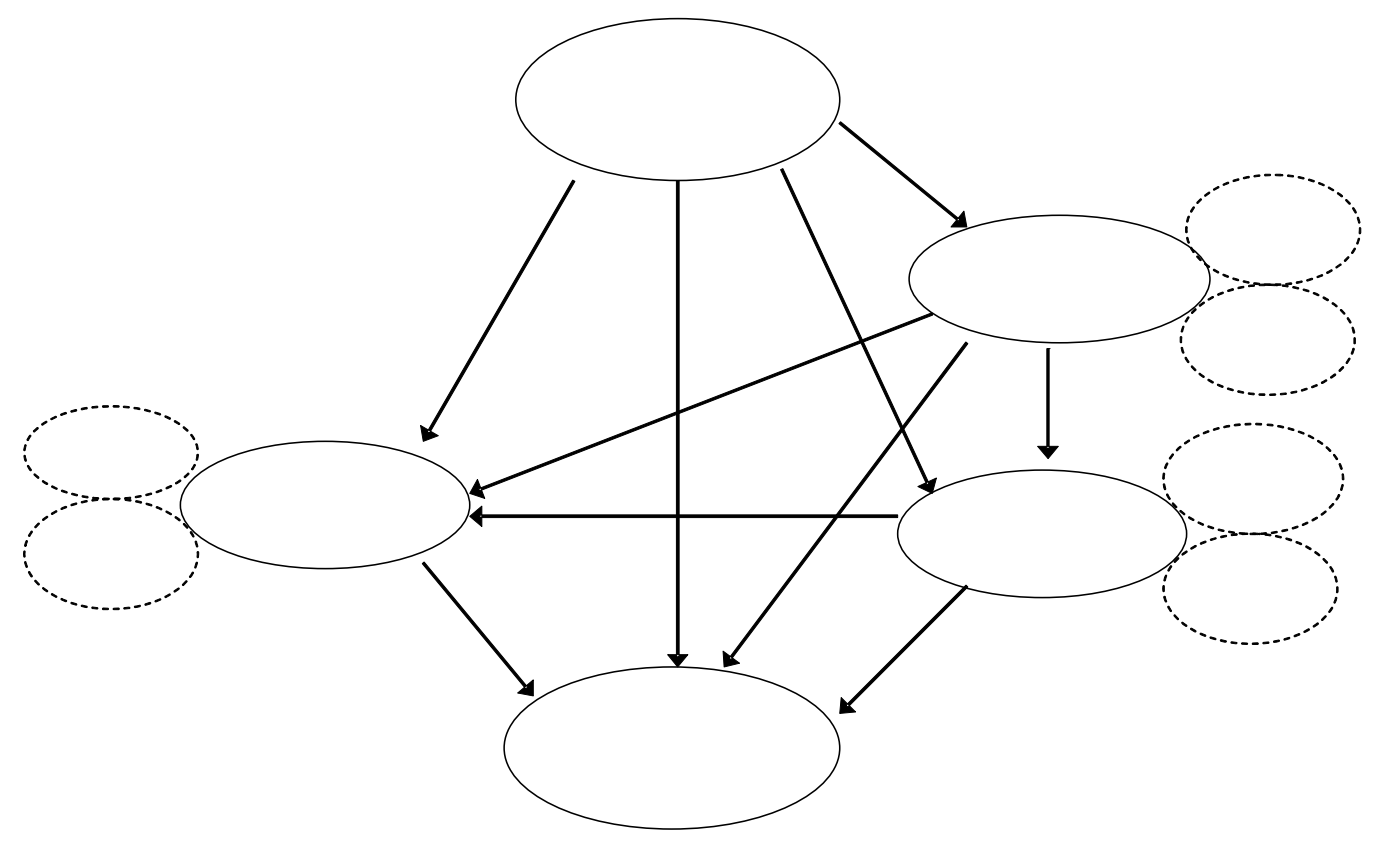

Figure 1. Simplified depiction of the post-acute health state transition process 


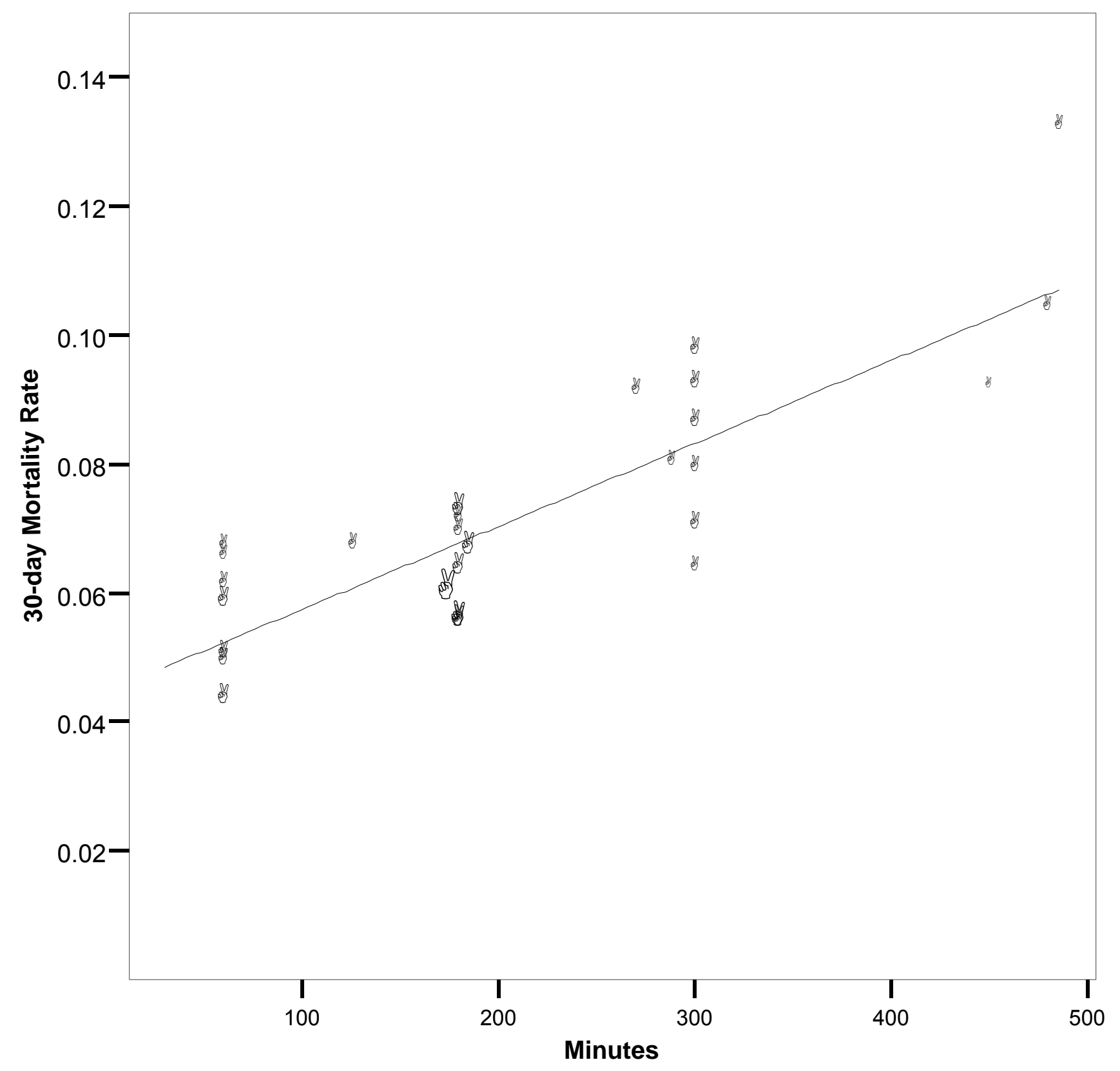

Figure 2. Time to needle and 30-day mortality (Circle size represents numbers of observations from a trial at each time point) 


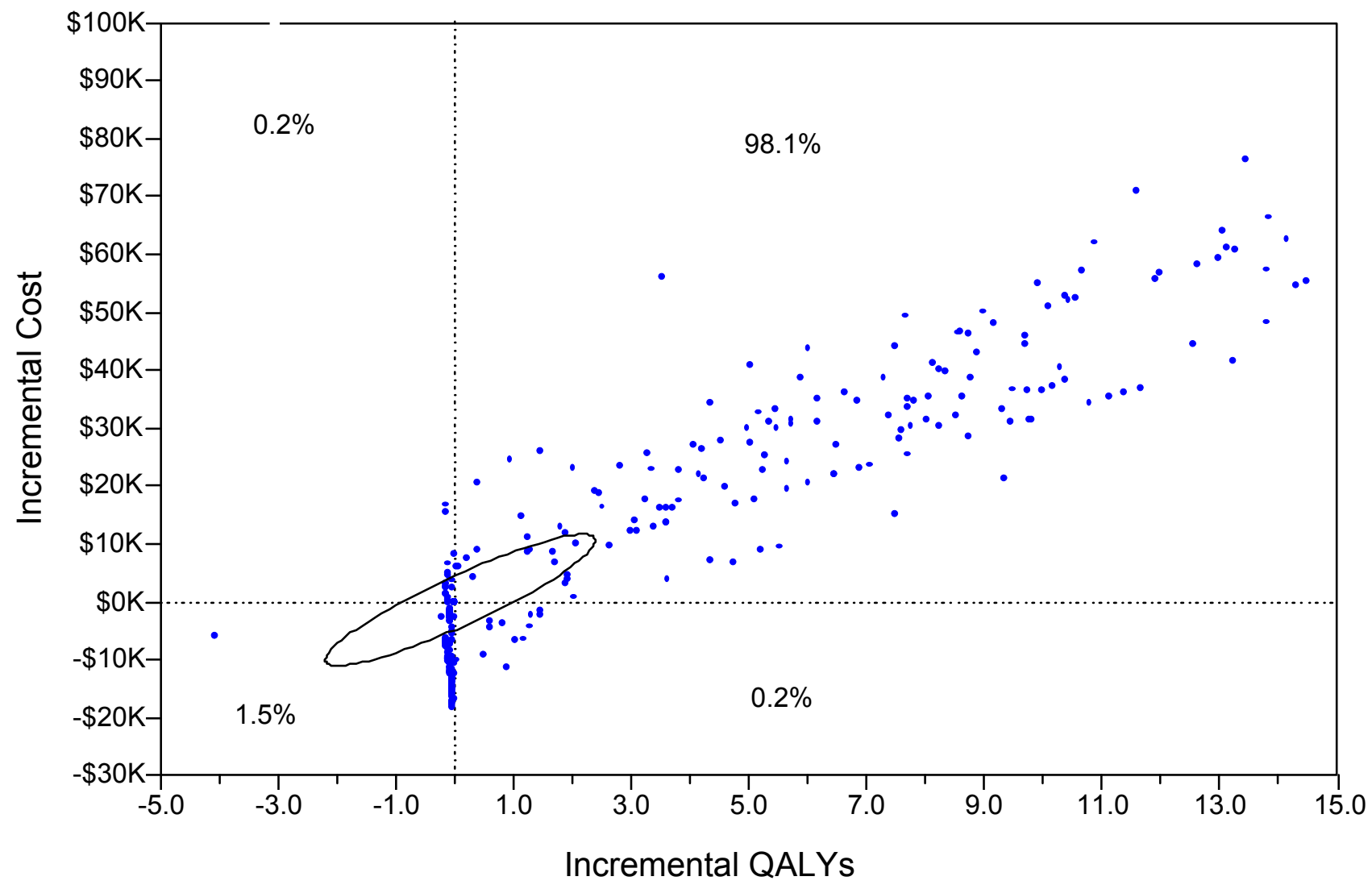

Figure 3. Scatterplot and $95 \%$ confidence ellipse of the Incremental Cost-Effectiveness

Ratios for the rest of life 


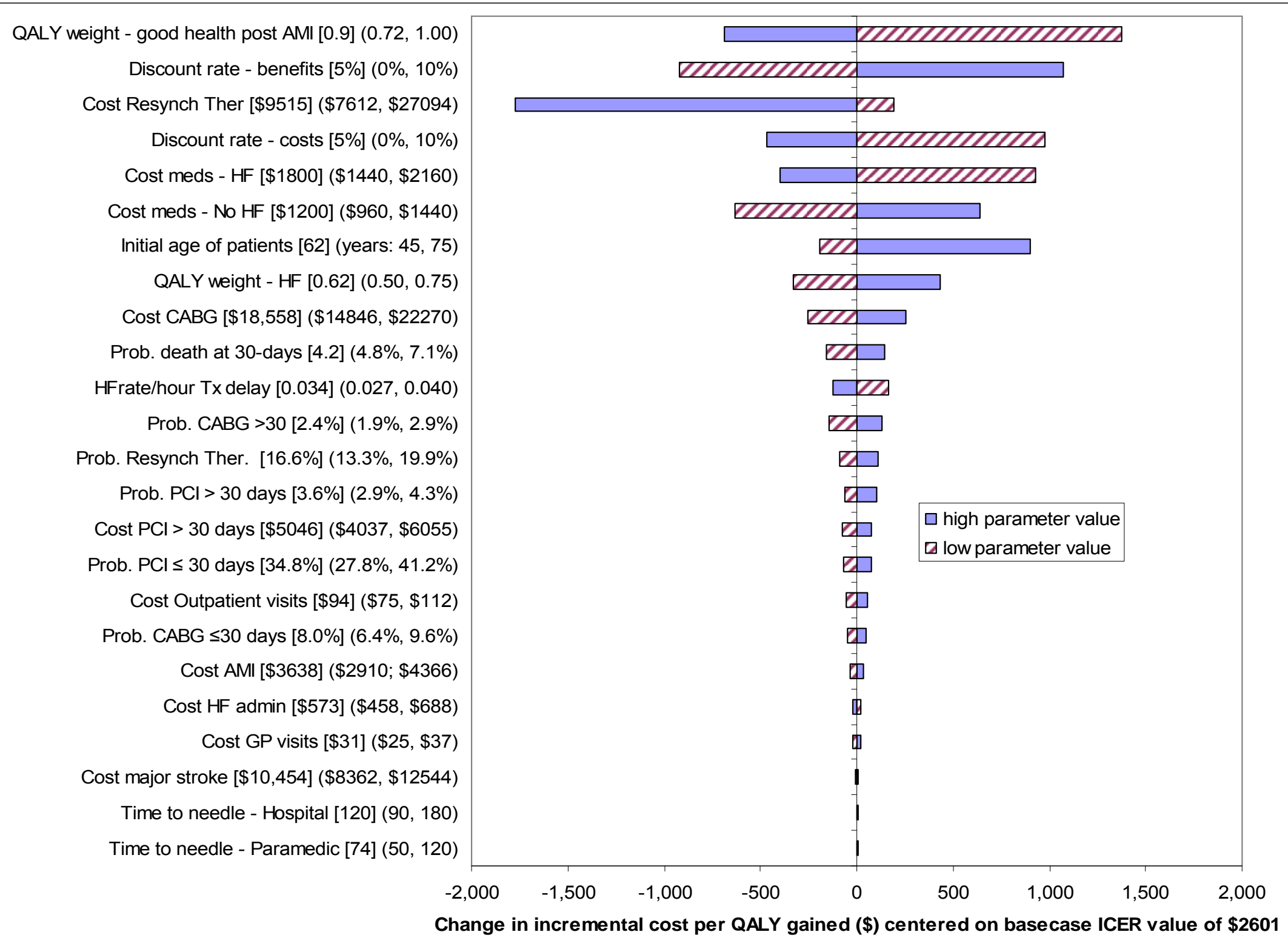

Figure 4. One-way sensitivity analysis (rest of life horizon). All costs are in 2004 A\$. 
